\title{
Human Activities - Their Classification, Recognition and Ensemble of Classifiers
}

\author{
Prajakta Kishore \\ Kalangutkar \\ Gogte Institute of Technology \\ Udyambag, Belgaum - 08 \\ Karnataka, India
}

\author{
Santosh S.Saraf \\ Gogte Institute of Technology \\ Udyambag, Belgaum - 08 \\ Karnataka, India
}

\author{
Sheetalkumar Mallikarjun \\ Onkari \\ KLE Dr.M.S.Sheshgiri College \\ of Engineering \& Technology \\ Udyambag, Belgaum - 08 \\ Karnataka, India
}

\begin{abstract}
Heart Rate Variability (HRV) testing aids in evaluating the body's ability to adapt to physical and mental stress. With the advent of computers, there are growing interests on recognizing context generated from human known as human activity recognition. The recognition of human activities has gained a higher interest in this field. Thus, this paper aims at recognizing the human activities by evaluating the HRV in real time, using a Pulse Sensor and by transferring the data serially from Arduino to MATLAB for classifying the activities and recognizing them. The paper also utilizes the data obtained from Wearable Action Recognition Database (WARD) database by creating a test data from the available dataset to implement the classifier algorithm.
\end{abstract}

\section{General Terms}

Human Activity Classification, Heart Rate Variability.

\section{Keywords}

heart rate variability; human activities; classification; feature extraction

\section{INTRODUCTION}

The advent of Microelectronics and computer systems has led to the development of various sensors and electronic devices which are capable of interacting with people as a part of their daily living. These developments have also led to advancements in the field of Biomedical engineering to help the doctors and caregiver to keep a track of the patients' behavior and responses. Thus recognition of human activities has gained a particular interest, especially for patients with diabetes, obesity or heart diseases as they are required to follow a well defined exercise routine.

Although the first work on recognition of human activities was carried out in late 90's, the technology for recognition of human activities needs to be improved, as some of the aspects are yet to be resolved completely. Some of these aspects are: (i) collection and selection of data set; (ii) implementation of feature extraction and classification algorithms; (iii) construction of a small, user friendly and portable system; (iv) collection of real-time data with minimum delay.
According to the studies on the functioning of the heart, it has been shown that, the period of heartbeat is not constant and changes over time with various human activities.

Variations in heart rate and their respective periods are called heart rate variability (HRV). The HRV obtained from longterm and short-term interval has been widely used to analyze the autonomic functions in patients with neurological dysfunction, cardiovascular diseases, diabetes, etc. Thus incorporating a more detailed information of the heart rate variability would be beneficial for interpreting the role of physical activities.

Day-by-day as the humans and computers are converging into an inseparable entity, the computers have gained an ability to recognize and understand its users dynamically, by obtaining the current and updated information about the user. Moreover, the advances in serial communication has led to an easy and quick transfer of the HRV data from a sensor with the help of a Microcontroller which can be further processed using various software processes in order to recognize the activities and classify them based on the HRV.

\section{LITERATURE REVIEW}

Hsiao-Lung Chan, et al. (2006: pp. 133-139) mentioned in their paper that the period of heartbeat is not constant and changes over time. Variations in heart rates and their respective periods are called heart rate variability (HRV). They have incorporated discrete wavelet transform to retrieve time-varying characteristics of heart rate variability under different physical activities. Their results showed that the heartbeat fluctuations in higher frequencies were greatest during lying and smallest during standing.

O'scar D. Lara and Miguel A. Labrador claimed that despite human activity recognition (HAR) being an active field for more than a decade, there are still key aspects that, if addressed, would constitute a significant turn in the way people interact with mobile devices. Their paper surveyed the state of the art in HAR based on wearable sensors.

David Naranjo-Hern’andez, et al. (2012: pp. 3177-3184) presented the hardware and software design and implementation of a low-cost, wearable, and un-obstructive intelligent accelerometer sensor for the monitoring of human 
physical activities. Their results have demonstrated the feasibility of the prototype and the proposed algorithms.

Liming Chen, et al. (2012: pp. 961-974) introduced in their paper a knowledge-driven approach to real-time, continuous activity recognition based on multisensor data streams in smart homes. Their proposed approach was implemented in a function-rich software system, which was deployed in a smart home research laboratory. They evaluated the proposed approach and the developed system through extensive experiments involving a number of various $\mathrm{ADL}$ use scenarios.

Al-Busaidi, A.M. described in his paper the development of innovative low-cost educational platform to study and control a biped robot in real-time using MATLAB and Arduino board. MATLAB was used as a control and visualization environment, while the Arduino board was utilized as a final controller for the servo motors as well as a Data Acquisition Card (DAC).

\section{IMPLEMENTATION}

The proposed implementation model mainly consisted of three blocks as shown in Fig. 1.

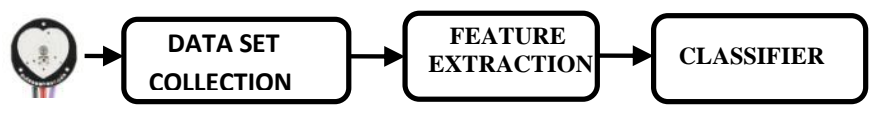

Fig.1. Block diagram of the proposed implementation model

Data Set Collection: It consisted of gathering the data from particular set of people of varying ages who are asked to perform some activities, using a heart rate sensor for a particular duration. In this project, data collection is done by two methods:

- Data obtained from pulse sensor.

- Data from the available data set.

Feature Extraction: It consisted of extracting the Heart Rate Variability (HRV) and Beats per Minute (BPM) by calculating the interbeat Interval.

Classifiers: It consisted of classifying the activities of various subjects based on their HRV analysis.

Fig. 2 shows a more detailed diagram of the proposed implementation model.

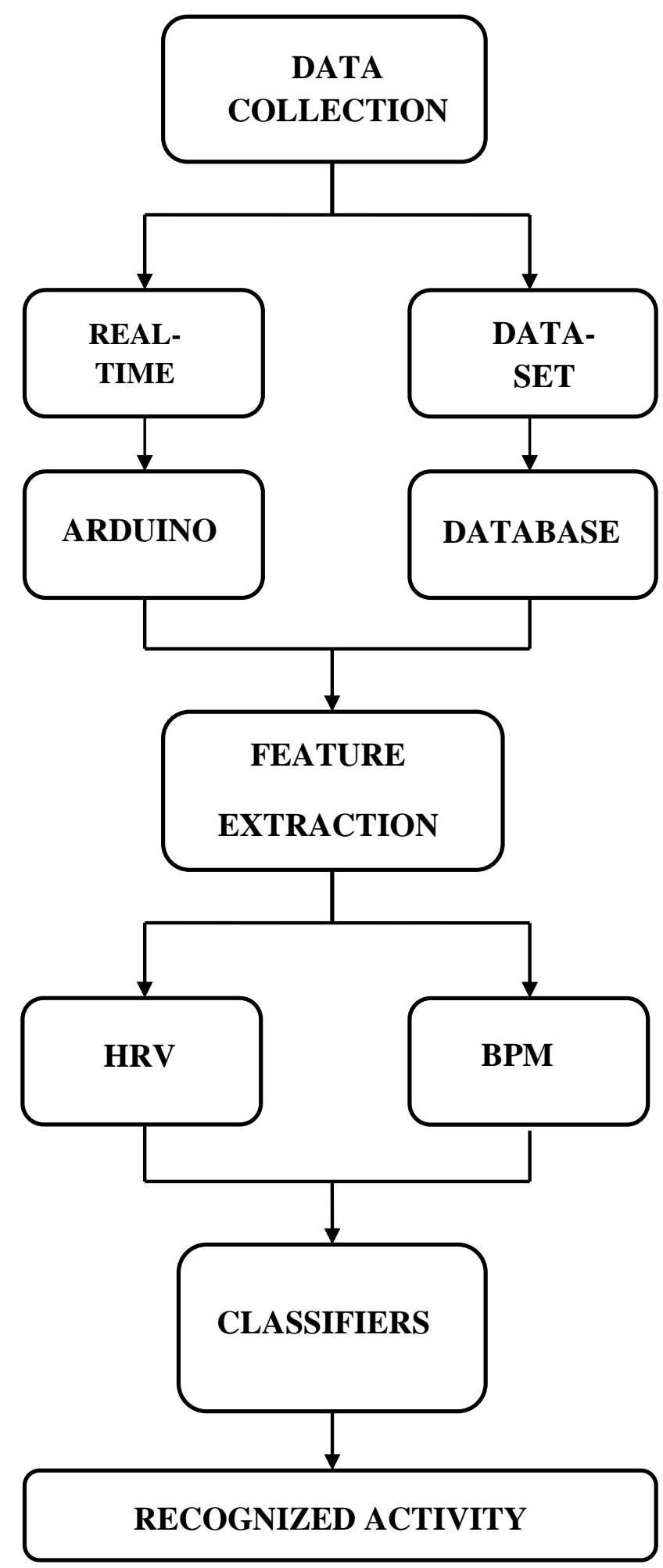

Fig.2. A more detailed block diagram of the proposed implementation model 


\subsection{Data Set Collection}

\subsubsection{Data Obtained from Pulse Sensor (Real- Time):}

The data set is collected in real-time using a Pulse Sensor Amped by Joel Murphy and Yury Gitman for Arduino [14]. This Pulse Sensor is essentially a photoplethysmograph, which is a well known medical device used for non-invasive heart rate monitoring. The data from this pulse sensor was extracted using Arduino [17], which is a single-board microcontroller and can be graphically visualized using Processing [18], which is an open source programming language and integrated development environment (IDE) built for the electronic arts, new media art, and visual design communities with the purpose of teaching the fundamentals of computer programming in a visual context, and to serve as the foundation for electronic sketchbooks [19]. The data from the Arduino Microcontroller was further transferred to MATLAB via serial communication [20], as it has an interactive development and debugging capabilities.

\subsubsection{Data from the available Data Set:}

The data set used in the project was from the Wearable Action Recognition Database (WARD), Version1.0, which served as a baseline benchmark [11]. The WARD data format uses the MATLAB environment to store the action data sampled from the wearable sensors. Each action sequence is given a trial number $\mathrm{N}, \mathrm{N}=1,2 \ldots$ The file name for storing the action data for the Nth trial is: WearableAction $<\mathrm{N}>$. mat, where < $\mathrm{N}>$ is to be replaced with the trial number. Each trial file has complete sensor readings stored of the action sequence in one variable: WearableData. Wearable-Data contains the following fields:

i. Version: A string indicating the version of the database. Currently the only value is: "WARD 1.0."

ii. Date: A string indicating the date of the recording session. The string has the format: "yyyy-mm-dd".

iii. Class: An integer value representing the action class number.

iv. Subject: An integer value representing the subject number.

v. Age: An integer value representing the age of the subject.

\subsection{Feature Extraction}

\subsubsection{ExtractingFeatures from Sensor and transferring it to MATLAB (Real-Time):}

The main features to be extracted are the Heart Rate Variability (HRV) and the Beats per minute (BPM). This is done using the pulse sensor and the principle given in [21]. The heart pulse signal that comes out of a photoplethysmograph is an analog fluctuation in voltage, and it has a predictable wave shape [21]. The depiction of the pulse wave is called a photoplethysmogram, or PPG. The latest hardware version, Pulse Sensor Amped, amplifies the raw signal of the previous Pulse Sensor, and normalizes the pulse wave around V/2 (midpoint in voltage). Pulse Sensor
Amped responds to relative changes in light intensity. If the amount of light incident on the sensor remains constant, the signal value will remain at 512 (midpoint of ADC range). More the light and the signal go up. Less the light, the signal goes down. Light from the LED, that is reflected back to the sensor changes during each pulse. The goal is to find successive moments of instantaneous heart beat and measure the time between, called the Inter Beat Interval (IBI). Once the pulse is detected and the heart rate variability (HRV) and the beats per minute (BPM) are calculated, the data is transferred serially to MTALAB [16], as shown in fig. 3 .

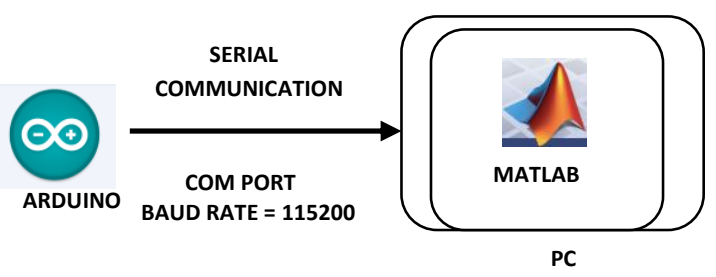

Fig. 3. Block diagram depicting Serial Communication between Arduino \& MATLAB

\subsubsection{Extracting features from the available WARD Data Set:}

The following features were extracted from each of the data set [6] available from the WARD data [11].

- Mean

- $\quad$ Standard Deviation

- Energy

- Correlation

The mean value represents the DC component of the signal over the window time frame while the standard deviation allows for the discrimination of similar data values for differing activities. Energy is a measure of the intensity of movement and is calculated by taking the sum of the squared discrete FFT magnitudes and dividing by the window length. Correlation enables the differentiation of activities that involve transition, i.e. between walking, running and stair climbing [6]

\subsection{Classifiers}

\subsubsection{Classifying the Activities from the Features Extracted in Real-Time:}

Although many classification algorithms have been proposed to classify human activities based on features extracted, in this project an attempt has been made to classify these activities empirically by recording the HRV of five subjects performing two different activities using PLX-DAQ, which is software for obtaining the data extracted from any Microcontroller into Microsoft Excel [22]. The real time HRV data which was transferred serially to MATLAB was passed through a classifier function to recognize the activity. The implementation of this classifier is illustrated in the flowchart of fig. 4. 


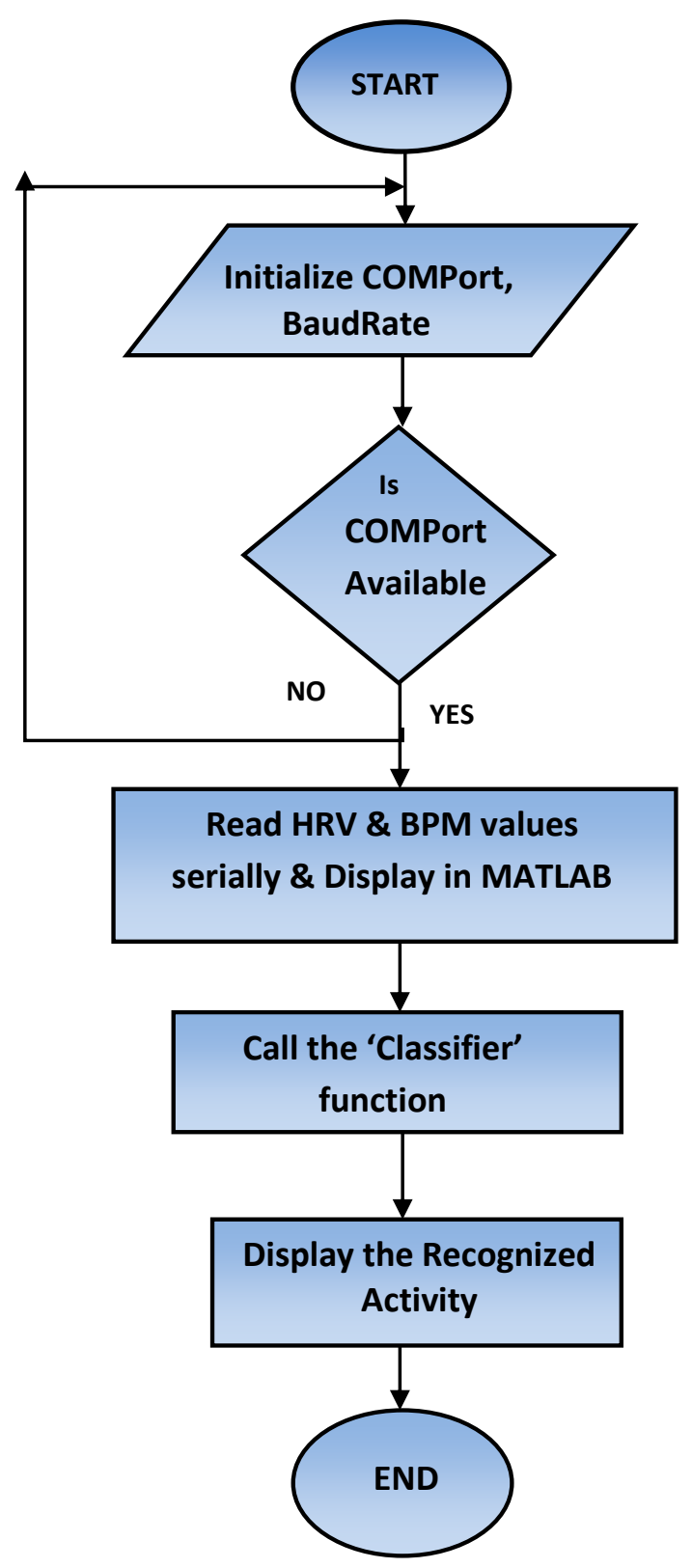

Fig. 4. Flowchart illustrating the implementation of Classifier algorithm for Recognizing Activity

\subsubsection{Classifying the Activities based on WARD Data Set:}

The data from WARD data set was divided into a training set and testing set. The aim was to make the user select the desired subject and the activity and train the classifier to extract single or all of the features of the available data set. The testing was then performed by selecting subject and the activity. The algorithm then classifies and displays the actual activity and the classified activity based on the training data set. The flowchart is as shown in fig.5.

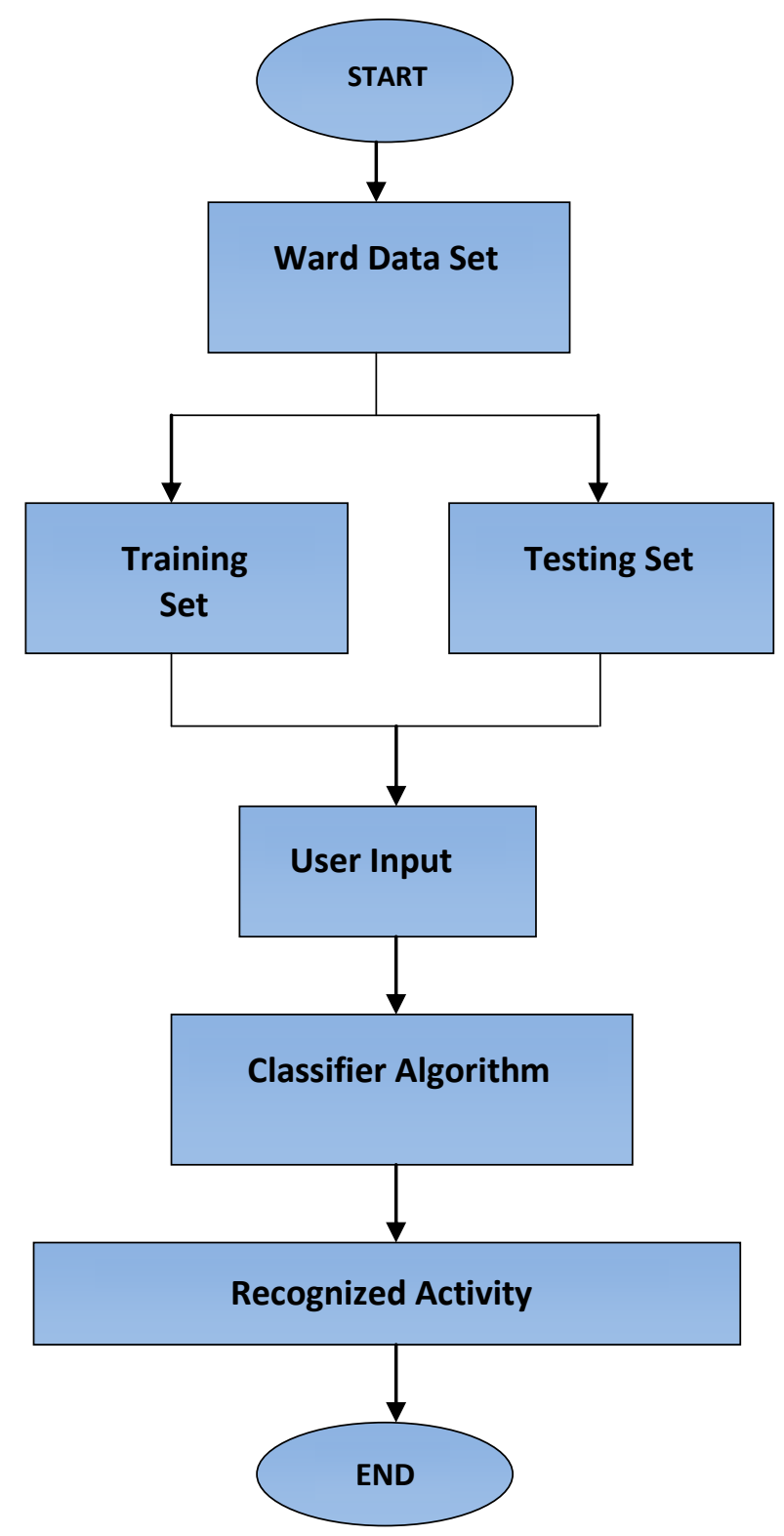

Fig. 5. Flowchart illustrating Classification of WARD Data set for Recognizing Activity

\section{RESULTS \& DISCUSSION}

The classification and recognition was done separately using the real time data and the WARD data set.

\subsection{Results obtained from the Real-Time Data}

The result obtained on the serial monitor of Arduino can be graphically visualized using Processing software. Since the data is a continuous time-varying data, the data was stored into an excel sheet, using the PLX-DAQ for training the algorithm. Table 1 shows the average HRV of 5 subjects, obtained from the Pulse Sensor using PLX-DAQ. 
TABLE 1. Average HRV recorded for 5 Subjects for two activities

\begin{tabular}{|l|l|l|}
\hline & Sitting & Standing \\
\hline Subject 1 & 708 & 635 \\
\hline Subject 2 & 706 & 620 \\
\hline Subject 3 & 755 & 625 \\
\hline Subject 4 & 746 & 607 \\
\hline Subject 5 & 756 & 605 \\
\hline
\end{tabular}

Using this data, the classifier algorithm was trained giving a result with a fair accuracy of around $85 \%$ as the algorithm is based on empirical logic. However due to the baud rate and serial communication issues, there can be a delay in obtaining the data.

\subsection{Results obtained from WARD Data Set}

The accuracy obtained from the WARD data base mainly depends on the training records. However in this project, since the test data is obtained from the given WARD database, the accuracy is around $95 \%$.

Table 2 shows the various feature extraction parameters and the accuracy obtained from the real time data and the WARD data set.

TABLE 2. Various parameters \& \%age accuracy obtained by real time data $\&$ the WARD dataset

\begin{tabular}{|l|c|c|}
\hline & $\begin{array}{c}\text { Real-Time } \\
\text { Data }\end{array}$ & $\begin{array}{c}\text { WARD } \\
\text { Database }\end{array}$ \\
\hline Mean & 751.3 & 1.2 \\
\hline Std. Deviation & 86 & 5 \\
\hline $\begin{array}{l}\text { Correlation } \\
\text { Coefficient }\end{array}$ & 0.075 & 0.04 \\
\hline Energy & 2.2 & 3.05 \\
\hline Accuracy & $85 \%$ & $95 \%$ \\
\hline Delay & $\begin{array}{c}\text { depends on } \\
\text { baud rate }\end{array}$ & minimum \\
\hline
\end{tabular}

\section{CONCLUSION \& FUTURE SCOPE}

Heart Rate Variability (HRV) testing evaluates the body's ability to adapt to physical and mental stress. Thus this work has been carried out to recognize the activities by evaluating the HRV in real time and recognizing the human activities which has become a task of high interest within the field, especially for medical, military, and security applications. Further, the data obtained from WARDs also utilized and the classifier algorithm was implemented to recognize the activity performed by using the training and test data.

However the delay due to serial communication between the Arduino and MATLAB tends to decrease the accuracy of the classifier algorithm. Thus the advances made so far need to be improved in terms of their robustness to real-world conditions and real-time performance.

\section{ACKNOWLEDGMENTS}

Our special thanks to the experts who have contributed towards the success of this study.

\section{REFERENCES}

[1] Hsiao-Lung Chan, Shih-Chin Fang, Yu-Lin Ko, HuiHsun Huang, and Chun-Hsien Lin, "Heart rate variability characterization in daily physical activities using wavelet analysis and multilayer fuzzy activity clustering" IEEE Transactions on Biomedical Engineering, vol.53,no.1,pp.133-139,Jan.2006.

[2] Mohd Fikri Azli bin Abdullah, Ali Fahmi Perwira Negara, Md. Shohel Sayeed, Deok-Jai Choi, Kalaiarasi Sonai Muthu, "Classification algorithms in human activity recognition using smartphones" World Academy of Science, Engineering and Technology 68 2012, pp. $422-429$

[3] Emmanuel Munguia Tapia, Stephen S. Intille,William Haskell, Kent Larson, Julie Wright, Abby King, and Robert Friedman "Real-Time recognition of physical activities and their intensities using wireless accelerometers and a heart rate monitor.'

[4] O' scar D. Lara and Miguel A. Labrador Department of Computer Science and Engineering University of South Florida, Tampa, FL 33620 "A survey on human activity recognition using wearable sensors."

[5] David Naranjo-Hern’andez, Laura M. Roa, Javier ReinaTosinaand Miguel A' ngel Estudillo-Valderrama," SoM: A smart sensor for human activity monitoring and assisted healthy ageing" IEEE transactions on biomedical engineering, vol. 59, no. 11, pp.3177 3183, Nov.2012.

[6] Maguire, D., Frisby, R. "Comparison of feature classification algorithm for activity recognition based on accelerometer and heart rate data", 9th. IT \& T Conference, Dublin Institute of Technology, Dublin, Ireland, 22nd.-23rd. October, 2009.

[7] Wansuree Massagram, Noah Hafner, Mingqi Cehn, Luca Macchiarulo, Victor M.Lubecke, and Olga BoricLubecke, "Digital heart-rate variability parameter monitoring and assessment ASIC", IEEE Transactions 
on Biomedical Circuits and Systems, vol.4, no.1,pp. 1926,Feb. 2010

[8] Liming Chen, Chris D. Nugent, Hui Wang, "A knowledge-driven approach to activity recognition in smart homes," IEEE Transactions on Knowledge and Data Engineering, vol. 24, no. 6, pp. 961-974, June 2012.

[9] Dipali Bansal, Munna Khan, A.K. Salhan, "A review of measurement and analysis of heart rate variability," iccae, pp.243-246, International Conference on Computer and Automation Engineering (iccae 2009), 2009.

[10] Al-Busaidi, A.M. ; Dept. of Mech. \& Ind. Eng., Sultan Qaboos Univ., Muscat, Oman, "Development of an educational environment for online control of a biped robot using MATLAB and Arduino", Published in Mechatronics (MECATRONICS) , $20129^{\text {th }}$ FranceJapan \& 7th Europe-Asia Congress on and Research and Education in Mechatronics (REM), 2012.

[11] Allen Y. Yang, "Data Collection Protocol for Wearable Action Recognition Database (WARD)."

[12] Heart Rate Variability Analysis Scientific Background$\mathrm{http} / / /$ www.biocomtech.com/hrvscientific, visited on 19/5/2013.

[13] Heart Rate Variability Analyzer http://zone.ni.com/reference/en-XX/help/373698A01/bioapps/hrv_analyzer/, visited on 19/5/2013.
[14] Pulse Sensor - http://www.pulsesensor.com/, visited on 29/4/2013.

[15] Introduction to Data Acquisition and Processing http://www.seas.upenn.edu/ ese111/Fall2012labs/Lab5.p df, visited on 1/5/2013.

[16] Arduino to MATLAB - read in sensor data http://robotgrrl.com/blog/2010/01/15/arduino-to-matlabread-in-sensor-data/, visited on 9/5/2013.

[17] Download software / Purchase Arduino www.arduino.cc/, visited on 11/4/2013.

[18] Download Processing with examples http://www.processing.org/, visited on 29/4/2013.

[19] Processing (Programming language) from Wikipedia, the free encyclopedia http://en.wikipedia.org/wiki/Processing_\%28programmin g_language\%29, visited on 30/4/2013.

[20] Serial Communication between Arduino and MATLAB http://home.iitb.ac.in/ rahul./ITSP/serial_comm_matlab. pdf, visited on $1 / 5 / 2013$.

[21] Pulse Sensor Amped Code \& Guide http://pulsesensor.myshopify.com/pages/pulse-sensoramped-arduino-v1dot1, visited on 6/5/2013.

[22] PLX-DAQ Data Acquisition for Excel http://www.parallax.com/ProductInfo/Microcontrollers/P LXDAQDataAcquisitiontool/tabid/393/Default.aspx, visited on 20/6/2013. 